\title{
AUTHORIAL STANCE IN COUNSELING RESEARCH ARTICLES
}

\author{
Abraham Cazares-Cervantes ${ }^{1}$, April B. LaGue, and Cass Dykeman \\ Oregon State University
}

\begin{abstract}
A popular notion is that authors in academic research articles merely convey propositional information. However, as Hyland has shown, authors in academic writing also use a range of devices to organize their texts, engage readers, and signal their attitudes to both their material and their readers (Hyland \& Tse, 2004). In the present study, three counselor educators examined the use of the devices of Self-Mentions, Boosters, Attitude Markers, and Hedges within a stratified random selection of research articles from 24 peer-reviewed counseling journals. Compared to a reference corpus of social science articles, counseling journals contained a greater use of self-mentions, attitude markers, and hedges. Implications for writers submitting their research to peer-reviewed counseling journals were presented. Keywords: counseling, authorial stance, hedges, boosters, corpus linguistics
\end{abstract}

\section{Introduction}

Novice academic writers view their efforts as strictly the conveyance of propositional information. Consideration of how their writing communicates a stance toward this information does not occur. This lack of contemplation takes place despite the fact that stance activities shape the gravitas (or lack thereof) of what is being communicated. As such, authorial stance practices in Counselor Education academic writing is the focus of the present study.

In a review of authorial stance in academic writing, six key topics emerged. These topics were: (1) definition of authorial stance, (2) function of hedges, (3) function of boosters, (4) function of attitude markers, (5) function of self-mentions, and (6) use rates of authorial stance markers across academic disciplines. After these six themes are reviewed, the research questions that guided the present study will be stated.

Many researchers have studied the forms in which writers express judgments, opinions and/or attitudes in their writing (Hyland, 2005; Hyland \& Tse, 2004). Authorial stance is one of the terms used to investigate this type of expressive writing. Authorial stance refers to the way in which writers present themselves and communicate their

1. Correspondence concerning this article should be addressed to Abraham CazaresCervantes, OSU Counseling Academic Unit, abraham.cazares-cervantes@oregonstate.edu 
personal authority about the topic using their feelings, concerns or judgements. In terms of the present study, we conducted a corpus linguistic study to explore the use of authorial stance in those articles from multiple journals.

Another topic this study explores is the use of hedges and boosters in the writing of social science research articles. Hedges are identified as language that withholds the writer's full commitment to a statement (Hyland, 2017). Hedges provide the writer with the tool to express uncertainty about claims and open the opportunity to other options. Some examples of hedges used in our study are: apparently, appears, assume, broadly, estimated, and feels. Boosters, on the other hand, are used by the writer to express certainty and emphasize the force of propositions (Hyland, 2017). Writers use boosters to express conviction about the written topic as boosters provide writers with the opportunity of expressing confidence in their knowledge of the topic and minimizing the possibility of accepting other options. Some examples of boosters used in our study are: actually, always, evidently, finds, in fact, and never among others.

The writers' ability to express attitude to propositions, agreement, importance, obligation or frustration are identified as attitude markers (Hyland, 2017). Attitude markers are an important part of writing in that writers express their stand on a specific topic pulling readers into a direction of agreeing with their view. Examples of attitude markers are: agree, astonishing, correctly, essentially, expected, and importantly among others.

Self-mention is another writing factor explored in this research. Self-mention refers to the authors' uses of first-person pronouns in their academic writing such as I, my, we, the author, us, among others (Hyland, 2017). Creating a presence in writing is essential and even more important in research writing, which provides writers with the opportunity to create a discoursal self in the text and ties that to the authors' values and beliefs that are connected to their discipline (Hyland, 2005).

In general, authorial stance is the ability of the author to express their interpretation of the data, the importance of the topic, feelings, claims and concerns using multiple stance resources in their written language (Hyland, 2005). This research evaluates the stance written language markers across social science journals in the counseling profession utilizing counseling research articles to explore how similar or different they are. 
Four research questions related to published counseling research articles were developed to guide this study. The first question was: What is the use rate of boosters, hedges, attitude markers and self-mentions overall, and by qualitative and quantitative research method? The second questions was: Does the use rate of boosters, hedges, attitude markers and self-mentions differ between qualitative and quantitative studies? The third question was: Does the use rate of boosters, hedges, attitude markers and selfmentions differ between high and low impact journals? The fourth question was: Does the use rate of boosters, hedges, attitude markers and self-mentions differ from known norms for social science research?

\section{Method}

\section{Design}

This study utilized a synchronic corpus linguistic design (Weisser, 2016). The unit of analysis for the first and fourth research questions was single words. The unit of analysis for the second and third research questions was research articles. The corpora were research articles that appeared in counseling research journals. The variables were: hedge words, booster words, attitude marker words, self-mention words, type of research (i.e., Qualitative or Quantitative), and level of journal impact (i.e., High or Low). All variables were continuous except for type of research and level of journal impact which were nominal.

The second and third research questions focus on comparing groups. Therefore, the appropriate power analysis was an independent t-test. This a priori power analysis was completed by means of $\mathrm{G}^{*}$ Power (Faul, Erdfelder, Buchner, \& Lang, 2009). The effect size was drawn from a study exploring linguistic features of psychology research articles (Esfandiari \& Barbary, 2017). The input parameters were: (a) Test family- t-tests, (b) Statistical test Means: Difference between two independent means (two groups), (c) Type of power analysis- a priori: compute required sample size- given $\alpha$, power, and effect size, (d) $d=0.83$, (e) power $(1-\beta$ error probability) $=0.80,(\mathrm{f}) \alpha=.05$, (g) allocation ratio $\mathrm{N} 2 / \mathrm{N} 1-1$, and $(\mathrm{f})$ degrees of freedom $(D f)=46$. The $\mathrm{G}^{*}$ Power 3.1 output suggested a sample size for both groups of 24 with an actual power of 0.8036 .

The fourth research question involves comparing obtain proportions against the expected. As such, a power analysis was for a chi-square goodness of fit test was conducted. The proper effect size for such a test is Cohen's $w$ (Rosnow \& Rosenthal, 2003). The effect size was drawn from a study examining discourse styles in an academic 
setting (Sullivan, Kapur, Madden, \& Shipe, 2015). The input parameters were: (a) Test family- $\chi 2$ tests, (b) Statistical test- Goodness-of-fit tests: contingency tables, (c) Type of power analysis- a priori: compute required sample size- given $\alpha$, power, and effect size, (d) $w=0.60,(\mathrm{e})$ power $(1-\beta$ error probability) $=0.80$, (f) $\alpha=.05$, and $(\mathrm{g})$ degrees of freedom $(D f)=6$. The $\mathrm{G}^{*}$ Power 3.1 output suggested a sample size of 38 with an actual power of 0.80 .

\section{Corpus}

The corpus employed for this study was the Oregon State Corpus of Counselor Education Research Articles (OCCERA). The corpus contains 517,231 tokens from 94 randomly selected research articles that appeared in one of 24 peer-reviewed counseling journals. Details on the scope, size, and construction of this corpus can be obtained at LaGue, Cazares-Cervantes, and Dykeman (2019).

\section{Measures}

Hedges. Words that denote a writer withholding a full commitment to a proposition (Hyland, 2017). An example is the word "might."

Boosters. Words that emphasize the writer's force or certainty in proposition in fact (Hyland, 2017). An example is the word "definitely."

Attitude Markers. Words where the writer expresses an attitude to proposition (Hyland, 2017). An example is the word "surprisingly."

Self-Mentions. Words that contain an explicit reference to author (Hyland, 2017). An example is the word "my."

Quantitative Design. Articles that used inferential statistics were classified as Quantitative as long as there was not present some form of qualitative analysis.

Qualitative Design. If the article noted the use of one of the following methods it was classified as Qualitative: Ethnography, Grounded Theory, Phenomenology, Ethnomethodology, Case Study, and Action Research (Cohen \& Crabtree, 2006). The one exception was articles where the use of inferential statistics was present. 
Mixed Design. Articles that contained both inferential statistics and some form of qualitative methodology were coded Mixed. So few articles of this type were encountered $(n=2)$ that the OCCERA creators did not include this category in their corpus.

Level of Impact. Few counseling journals possess an ISI Impact factor score. As such, the Scimago H Index was used (Scimago Labs, 2019). A coding of "High" was operationalized as a journal possessing a Scimago H Index score above the median for all 24 journals and a coding of "Low" was operationalized as a journal possessing a score below the median.

Reference Corpus. The reference corpus was drawn from the average of the social science subcorpus created by Kwary (2018). This subcorpus contained 122 peer reviewed articles and 1,152,562 tokens.

\section{Apparatus}

To count tokens and stance markers, the Authorial Voice Analyzer 1.0 for Mac (AVA) was used (Yoon, 2017). The AVA was selected because it employs Hyland's stance marker categories and accounts for multi-functionality of words in an automated manner. This software produces raw counts for all four stance markers as well as overall tokens and types. In addition, the AVA provides the normalized frequency for each stance marker type of per 1,000 words.

\section{Data Analysis}

For the first research question, both raw counts and percentage of all words will be provided. In reference to the second and three research questions, a t-test will be used unless the Levene's Test of Equality of Variances is significant and then the Welch's t-test will be employed. For effect size, a Cohen's $d$ will be provided. In terms of the fourth research question, overall stance marker results will be compared against known norms using Log-Likelihood ( $L L$ ) analysis (Rayson \& Garside, 2000). The effect size measure reported will be the Bayesian Information Criterion (BIC) (Brezina, 2018). An alpha level for all inferential analyses was set at .05 and calculations will be completed using Excel and JASP (2019).

\section{Results}

Frequency counts for percentage of all words for each stance marker can be found in Table 1. Stance marker differences for research method and journal ranking can be examined in Table 2. In terms of differences between High and Low journal rankings, no 
statistical differences were encountered. Finally, Self-Mention, Attitude, Hedge marker

levels were higher than for the refence corpus of social science research articles (see Table $3)$.

\section{Discussion}

This study explored the use of authorial stance, hedges, boosters, attitude markers, and self-mentions among qualitative and quantitative research articles in counseling. Using a synchronic corpus linguistic design, we examined the differences between qualitative and quantitative articles in the use of language. The results of the first research question (use rate overall and by research method) demonstrated a high overall frequency use of hedges in both research methods at a $15 \%$ usage. Additionally, overall usage for boosters and attitude markers in both was 10\%. One possible explanation for this is that scholars using either research method publish articles that tend to create a certain ambiguity to engage their readers and open discussion as opposed to stating a certainty. An alternative explanation is that journal reviewers are more likely to accept research articles that utilize this type of language and point of view. Between the former and latter explanations, the latter is most probable given the strong influence reviewers exert on what gets published.

The second research question looks at the use rate of boosters, hedges, attitude markers and self-mentions between the two research methods. The results for the four types of stance markers varied widely.

The differences in booster use between the two methods had a medium effect size. Quantitative studies demonstrated a higher use of boosters than qualitative studies. Boosters provide the writer the opportunity to express certainty and/or emphasize a specific point in the writing. These could indicate that quantitative writers engage in higher use of booster language to accentuate what they want readers to believe creating a unique sense of certainty in the claims, taking the reader in the direction of their claims and creating a sense of professional confidence on the topics.

Researchers from both methodologies used hedges equally. This similarity reflects how both research methods equally use language that avoids commitment to propositions and displays uncertainty about the truth of statements in the writing (Hyland, 2005).

Findings indicated a small effect size difference in the use of attitude markers between quantitative and qualitative research articles. A probable explanation for the 
usage of attitude markers in both types of studies is the idea that researchers are trying to persuade their audience by emphasizing and guiding their perceptions about the topic being researched.

The largest effect size difference between the two methods was with self-mentions. Results revealed that qualitative research articles demonstrated a significantly higher use of self-mention words than quantitative research articles. One probable reason for this finding is that qualitative researchers want to be more personal using self-mentions as a tool to engage the reader and present themselves as a reliable colleague (Hyland, 2003). Another possible reason to explain the findings is that the researchers aim to demonstrate their confidence and would like to be acknowledged as experts in the field. Between the former and latter explanations, the former is most likely because of the centrality of self as a research tool in this paradigm (Patton, 1999).

The results from the third research question (stance marker type by journal impact level) found no differences between high and low impact journals with regard to boosters, hedges, attitude markers and self-mentions. Based on the results, both high and low impact journals engage in similar writing strategies to connect the reader, to help the reader interpret and evaluate the information and to persuade the reader towards the perceptions of the author as the author takes a stand demonstrating a professional authoritative voice (Hyland, 2017).

With regard to the fourth research question (stance use v. reference corpus), two possible reasons should be noted for the strong differences for self-mentions, attitude markers, and hedges. One possible explanation is that the scholarly culture in counseling has evolved such that it favors greater use of these three devices than the broader social science discourse community. An alternative explanation is that the nature of counseling research pulls for such word choices to a greater extent than other social science disciplines. Since there is no logical reason to postulate that counseling phenomena differ radically from the other social sciences, the former explanation is the most probable.

The corpus employed for this study was the Oregon State Corpus of Counselor Education Research Articles (OCCERA). Two limitations to this corpus should be considered. First, the OCCERA comprises articles from journals from 2018. More articles may reveal a larger variance in the aforementioned results. Second, only national journals were included in the corpus. This left a potential gap in data involving state and international publications. Future research may broaden the data set by including these categories of publications. 
Writers may be able to use this research to guide their writing practices for publication. Understanding the need to create a stance pattern in their writing that mirrors the culture of the counseling discourse community may lead to a higher manuscript acceptance rate for authors. Additionally, when authors understand the most powerful way to express the interpretation of their data, their information may be more accurately shared and disseminated. Understanding the intentionality of including boosters, hedges, attitude markers, and self-mentions in an author's writing could increase the awareness and efficacy of novice writers in counselor education. Although much remains to be done to better understand the linguistic use of self-mention, attitude, boosters and hedges in counseling education research, this study tried to shed some light into how counseling researchers use the devices in their writing. $\backslash$

\section{References}

Anthony, L. (2019). AntConc (Version 3.5.8) [Computer Software]. Tokyo, Japan: Waseda University. Available from http://www.laurenceanthony.net/software

Brezina, V. (2018). Statistics in corpus linguistics: A practical guide. Cambridge, UK: Cambridge University Press.

Cohen, D., \& Crabtree, B. (2006). Common research traditions. Princeton, NJ: Robert Wood Johnson Foundation. Retrieved from http://www.qualres.org/HomeComm3582.html

Esfandiari, R., \& Barbary, F. (2017). A contrastive corpus-driven study of lexical bundles between English writers and Persian writers in psychology research articles. Journal of English for Academic Purposes, 29, 21-42. https://doi.org/10.1016/j.jeap.2017.09.002

Faul, F., Erdfelder, E., Buchner, A., \& Lang, A.-G. (2009). Statistical power analyses using $\mathrm{G}^{*}$ Power 3.1: Tests for correlation and regression analyses. Behavior Research Methods, 41, 1149-1160. http://dx.doi.org/10.3758/BRM.41.4.1149

Hyland, K. (2000). Disciplinary discourses: Social interactions in academic writing. London: Longman.

Hyland, K. (2005). Stance and engagement: A model of interaction in academic discourse. Discourse Studies, 7, 173-192. https://doi.org/10.1177/1461445605050365

Hyland, K. (2017). Metadiscourse: what is it and where is it going? Journal of Pragmatics, 113, 16-29. https://doi.org/10.1016/j.pragma.2017.03.007

Hyland, K., \& Tse, P. (2004). Metadiscourse in academic writing: A reappraisal. Applied Linguistics, 25, 156-177. https://doi.org/10.1093/applin/25.2.156 
JASP Team (2018). JASP (Version 0.10.0)[Computer software].

Kwary, D. A. (2018). A corpus and a concordancer of academic journal articles. Data in Brief, 16, 94-100. http://doi.org/10.1016/j.dib.2017.11.023

LaGue, A., Cazares-Cervantes, A., and Dykeman, C. (2019). OCCERA: Oregon State Corpus of Counselor Education Research Articles (OCCERA). PsyArXiv. https://doi.org/10.31234/osf.io/qnsvm

McDonald, J. H. (2014). Handbook of biological statistics (3rd ed.). Sparky House Publishing, Baltimore, MD. Retrieved from http://www.biostathandbook.com/exactgof.html\#posthoc

Patton, M. Q. (1999). Enhancing the quality and credibility of qualitative analysis. Health Services Research, 34, 1189-1208.

Rayson, P., \& Garside, R. (2000). Comparing corpora using frequency profiling. In WCC '00 Proceedings of the workshop on Comparing corpora (Vol. Volume 9, pp. 1-6). http://doi.org/10.3115/1117729.1117730

Rosnow, R. L., \& Rosenthal, R. (2003). Effect sizes for experimenting psychologists. Canadian Journal of Experimental Psychology/Revue Canadienne de Psychologie Expérimentale, 57, 221-237. http://dx.doi.org/10.1037/h0087427.

Scimago Labs. (2019). Scimago journal rankings. Retrieved from https://www.scimagojr.com/journalrank.php

Sullivan, F. R., Kapur, M., Madden, S. \& Shipe, S. (2015). Exploring the role of 'Gendered' discourse styles in online science discussions. International Journal of Science Education, 37, 484-504. https://doi.org/10.1080/09500693.2014.994113

Weisser, M. (2016). Practical Corpus Linguistics: An Introduction to corpus-based language analysis. Malden, MA \& Oxford: Wiley-Blackwell.

Yoon, H. J. (2017). Textual voice elements and voice strength in EFL argumentative writing. Assessing Writing, 32, 72-84. https://doi.org/10.1016/j.asw.2017.02.002 
Table 1

Stance Categories Counts and Percentages

\begin{tabular}{lcccccc}
\hline \multirow{2}{*}{ Stance Category } & \multicolumn{2}{c}{ Overall } & \multicolumn{2}{c}{ Quantitative } & \multicolumn{2}{c}{ Qualitative } \\
& Raw & \% of All & Raw & \% of All & Raw & \% of All \\
& Count & Words & Count & Words & Count & Words \\
\hline Hedge & 8,364 & 15.74 & 4,825 & 15.91 & 3,539 & 15.46 \\
Booster & 5,560 & 10.23 & 2,855 & 9.33 & 2,705 & 11.69 \\
Attitude Marker & 5,468 & 10.45 & 3,336 & 11.10 & 2,132 & 9.40 \\
Self-Mention & 3,056 & 5.25 & 517 & 1.72 & 2,548 & 10.94 \\
\hline
\end{tabular}


Table 2

Quantitative v. Qualitative Results

\begin{tabular}{lccccc}
\hline \multicolumn{1}{c}{ Category } & t-test & $t$ & $p$ & ES & ES Descriptor \\
\hline Self-Mention & Welch & -6.10 & $<.001$ & -1.42 & Very Large \\
Booster & Student & -3.03 & 0.00 & -0.65 & Medium \\
Attitude & Student & 2.04 & 0.04 & 0.43 & Small \\
Hedge & Student & 0.50 & 0.62 & 0.11 & Trivial \\
\hline
\end{tabular}

Note. Effect size given is Cohen's $d$. 
Table 3

Stance Marker Comparison to Known Norms

\begin{tabular}{lccccccccc}
\hline \multicolumn{7}{c}{ Observed } & \multicolumn{2}{c}{ Expected } \\
Category & OCCERA & $\begin{array}{c}\text { Kwary } \\
\text { SocSci }\end{array}$ & OCCERA & $\begin{array}{c}\text { Kwary } \\
\text { SocSci }\end{array}$ & $\begin{array}{c}\text { Over/ } \\
\text { under- } \\
\text { use }\end{array}$ & LL & BIC & $\begin{array}{c}\text { BIC } \\
\text { Descriptor }\end{array}$ \\
\hline Self-M. & 3065 & 2197 & 1650 & 3612 & + & 1610.38 & 1596.05 & Very Strong \\
Attitude & 5468 & 9529 & 4704 & 10293 & + & 176.05 & 161.72 & Very Strong \\
Hedge & 8364 & 15709 & 7551 & 16522 & + & 125.31 & 110.98 & Very Strong \\
Booster & 5560 & 11484 & 5346 & 11698 & + & 12.39 & -1.94 & Trivial \\
\hline
\end{tabular}

